\title{
Beating the diffraction limit in astronomy via quantum cloning (Corrigendum)
}

\author{
A. Kellerer \\ Cavendish Laboratory, University of Cambridge, Cambridge CB3 OHE, UK \\ e-mail: ak935@cam.ac.uk
}

A\&A 561, A118 (2014), DOI: 10.1051/0004-6361/201322665

Key words. telescopes - instrumentation: high angular resolution - errata, addenda

In Sect. 3.3 of Kellerer (2014) I suggested detector read-out times below the coherence time of photons. If one assumes, instead, read-out times not less than the coherence time, $\Delta t=$ $\lambda^{2} /(c \Delta \lambda)$, the spontaneous photons exceed, even for a very small field-of-view, the number of stimulated photons per incoming photon.

With the notation employed in Kellerer (2014) the mean number of cloned photons per incoming photon is:

$N-1=\frac{\sigma}{S} I$

where $I$ is the number of excited atoms, $\sigma$ is the cross-section of excited atoms and $S$ is the aperture- and amplifier-area. A field of angular diameter $\theta=2.44 \lambda / D$ - where $D$ is the aperture diameter - corresponds to the Airy disc up to its first minimum. Within the read-out time $\Delta t=\lambda^{2} /(c \Delta \lambda)$, equal to the photon coherence time, this "diffraction area" receives a mean number of spontaneous photons:

$M=\frac{\pi \theta^{2}}{4} \cdot \frac{1}{4 \pi} \cdot A \Delta t$

$A$ is the spontaneous emission rate.

From these relations one obtains the average fluence ratio of spontaneous and stimulated photons on the diffraction area:

$\frac{M}{N-1}=0.74 \pi^{2} \sim 7.3$ in line with calculations by Prasad (1994) and his conclusions that the spontaneous emissions dominate the stimulated ones. On the other hand, on average $0.64 \mathrm{~N}$ cloned photons end up on the central standard deviation range of diameter $1 / 3$ of the Airy disc. This area is 9 times smaller than the Airy disc considered above. Thus the ratio of spontaneous to stimulated fluence is not $7.3 \mathrm{but}$ merely $7.3 /(9 \times 0.64) \sim 1.3$ in this region around the centre of the cloned photons.

The spontaneous photons will prevent a large improvement of resolution as long as our set-up lacks a stage to recognize events where the stimulated emissions dominate. Such a stage is in principle possible, see notably the probabilistic noiseless amplification processes discussed by Duan \& Guo (1998), Ralph \& Lund (2009). The main message of my article remains: it is fundamentally possible to improve the resolution of a telescope beyond the diffraction limit at the price of sensitivity, i.e. it is possible to trade sensitivity against resolution. The set-up that I have suggested will be incomplete unless it is given a suitable heralding stage.

\section{References}

Duan, L.-M., \& Guo, G.-C. 1998, Phys. Rev. Lett., 80, 4999 Kellerer, A. 2014, A\&A, 561, A118

Prasad, S. 1994, J. Optic. Soc. Am. A, 11, 2799

Ralph, T. C., \& Lund, A. P. 2009, in AIP Conf. Ser. 1110, ed. A. Lvovsky, 155 